\title{
For each $\alpha>2$ there is an infinite binary word with critical exponent $\alpha$
}

\author{
James D. Currie*\& Narad Rampersad ${ }^{\dagger}$ \\ Department of Mathematics and Statistics \\ University of Winnipeg \\ Winnipeg, Manitoba R3B 2E9 \\ CANADA \\ e-mail: j.currie@uwinnipeg.ca, \\ n.rampersad@uwinnipeg.ca
}

Submitted: Feb 28, 2008; Accepted: Aug 25, 2008; Published: Aug 31, 2008

Mathematics Subject Classification: 68R15

\begin{abstract}
The critical exponent of an infinite word $\mathbf{w}$ is the supremum of all rational numbers $\alpha$ such that $\mathbf{w}$ contains an $\alpha$-power. We resolve an open question of Krieger and Shallit by showing that for each $\alpha>2$ there is an infinite binary word with critical exponent $\alpha$.
\end{abstract}

Keywords: Combinatorics on words, repetitions, critical exponent

\section{Introduction}

If $\alpha$ is a rational number, a word $w$ is an $\alpha$-power if there exist words $x$ and $x^{\prime}$ and a positive integer $n$, with $x^{\prime}$ a prefix of $x$, such that $w=x^{n} x^{\prime}$ and $\alpha=n+\left|x^{\prime}\right| /|x|$. We refer to $|x|$ as a period of $w$. A word is $\alpha$-power-free if none of its subwords is a $\beta$-power with $\beta \geq \alpha$; otherwise, we say the word contains an $\alpha$-power.

The critical exponent of an infinite word $\mathbf{w}$ is defined as

$$
\sup \{\alpha \in \mathbb{Q} \mid \mathbf{w} \text { contains an } \alpha \text {-power }\} .
$$

Critical exponents of certain classes of infinite words, such as Sturmian words $[8,10]$ and words generated by iterated morphisms $[5,6]$, have received particular attention.

Krieger and Shallit [7] proved that for every real number $\alpha>1$, there is an infinite word with critical exponent $\alpha$. As $\alpha$ tends to 1, the number of letters required to construct

${ }^{*}$ The author's research was supported by an NSERC operating grant.

†The author is supported by an NSERC Post-doctoral Fellowship. 
such words tends to infinity. However, for $\alpha>7 / 3$, Shur [9] gave a construction over a binary alphabet. For $\alpha>2$, Krieger and Shallit gave a construction over a four-letter alphabet and left it as an open problem to determine if for every real number $\alpha \in(2,7 / 3]$, there is an infinite binary word with critical exponent $\alpha$. Currie, Rampersad, and Shallit [3] gave examples of such words for a dense subset of real numbers $\alpha$ in the interval $(2,7 / 3]$. In this note we resolve the question completely by demonstrating that for every real number $\alpha>2$, there is an infinite binary word with critical exponent $\alpha$.

\section{Properties of the Thue-Morse morphism}

In this section we present some useful properties of the Thue-Morse morphism; i.e., the morphism $\mu$ defined by $\mu(0)=01$ and $\mu(1)=10$. Note that $\left|\mu^{s}(0)\right|=\left|\mu^{s}(1)\right|=2^{s}$ for all $s \geq 0$.

Lemma 1. Let $s$ be a positive integer. Let $z$ be a subword of $\mu^{s+1}(0)=\mu^{s}(01)$ with $|z| \geq 2^{s}$. Then $z$ does not have period $2^{s}$.

Proof. Write $\mu^{s}(0)=a_{1} a_{2} \ldots a_{2^{n}}, \mu^{s}(1)=b_{1} b_{2} \ldots b_{2^{n}}$. One checks by induction that $a_{i}=1-b_{i}$ for $1 \leq i \leq 2^{n}$, and the result follows.

Brandenburg [1] proved the following useful theorem, which was independently rediscovered by Shur [9].

Theorem 2 (Brandenburg; Shur). Let $w$ be a binary word and let $\alpha>2$ be a real number. Then $w$ is $\alpha$-power-free if and only if $\mu(w)$ is $\alpha$-power-free.

The following sharper version of one direction of this theorem (implicit in [4]) is also useful.

Theorem 3. Suppose $\mu(w)$ contains a subword $u$ of period $p$, with $|u| / p>2$. Then $w$ contains a subword $v$ of length $\lceil|u| / 2\rceil$ and period $p / 2$.

We will also have call to use the deletion operator $\delta$ which removes the first (left-most) letter of a word. For example, $\delta(12345)=2345$.

\section{$3 \quad$ A binary word with critical exponent $\alpha$}

We denote by $\mathcal{L}$ the set of factors (subwords) of words of $\mu\left(\{0,1\}^{*}\right)$.

Lemma 4. Let $00 v \in \mathcal{L}$, and suppose that $00 v$ is $\alpha$-power-free for some fixed $\alpha>2$. Let $r=\lceil\alpha\rceil$. Suppose that $0^{r} v=x u y$ where $u$ contains an $\alpha$-power. Then $x=\epsilon$ and $u=0^{r}$.

Proof. Suppose that $u$ has period $p$. Since $00 v \in \mathcal{L}, v$ begins with 1 . Since $00 v$ is $\alpha$ power-free, we can write $u=0^{s} v^{\prime}$, where $x=0^{r-s}$ for some integer $s, 3 \leq s \leq r$, and $v^{\prime}$ is a prefix of $v$. If $0^{p}$ is not a prefix of $u$ then the prefix of $u$ of length $p$ contains the 
subword 0001. Since $\alpha>2$, this means that 0001 is a subword of $u$ at least twice, so that 0001 is a subword of $00 v$. This is impossible, since $00 v \in \mathcal{L}$.

Therefore, $0^{p}$ is a prefix of $u$, and $u$ has the form $0^{t}$ for some integer $t \geq \alpha$. This implies that $u$ has $0^{r}$ as a prefix, so that $x=\epsilon$ and $u=0^{r}$.

Lemma 5. Let $\alpha>2$ be given, and let $r=\lceil\alpha\rceil$. Let $s, t$ be positive integers, such that $s \geq 3$ and there are words $x, y \in\{0,1\}^{*}$ such that $\mu^{s}(0)=x 00 y$ with $|x|=t$. Suppose that $2<r-t / 2^{s}<\alpha$ and $00 v \in \mathcal{L}$ is $\alpha$-power-free. Then the following statements hold.

1. The word $\delta^{t} \mu^{s}\left(0^{r} v\right)$ has a prefix which is a $\beta$-power, where $\beta=r-t / 2^{s}$.

2. Suppose that $00 v$ contains a $\beta$-power of period $p$ for some $\beta$ and $p$. Then $\delta^{t} \mu^{s}\left(0^{r} v\right)$ contains a $\beta$-power of period $2^{s} p$.

3. The word $\delta^{t} \mu^{s}\left(0^{r} v\right)$ is $\alpha$-power-free.

Proof. We start by observing that $\mu^{s}\left(0^{r}\right)$ has period $2^{s}$. It follows that $\delta^{t} \mu^{s}\left(0^{r}\right)$ is a word of length $r 2^{s}-t$ with period $2^{s}$, and hence is a $\left(r 2^{s}-t\right) / 2^{s}=\beta$-power.

Now suppose $u$ is a $\beta$-power of period $p$ in $00 v$. Then $\mu^{s}(u)$ is a $\beta$-power of period $2^{s} p$ in $\mu^{s}(00 v)$. However, $\mu^{s}\left(0^{r-1} v\right)$ is a suffix of $\delta^{t} \mu^{s}\left(0^{r} v\right)$, since $t<2^{s}=\left|\mu^{s}(0)\right|$. Thus $\mu^{s}(u)$ is a $\beta$-power of period $2^{s} p$ in $\delta^{t} \mu^{s}\left(0^{r} v\right)$.

Next, note that $\mu^{s}\left(0^{r-1} v\right)$ does not contain any $\kappa$-power, $\kappa \geq \alpha$. Otherwise, by Theorem 3 and induction, $0^{r-1} v$ contains a $\kappa$-power. This is impossible by Lemma 4 .

Suppose then that $\delta^{t} \mu^{s}\left(0^{r} v\right)$ contains a $\kappa$-power $\hat{u}$ of period $q, \kappa \geq \alpha$. Using induction and Theorem $3,0^{r} v$ contains a $\kappa$-power $u$ of period $q / 2^{s}$. By Lemma 4 , the only possibility is $u=0^{r}$, and $q / 2^{s}=1$. Thus $q=2^{s}$.

Since $00 v \in \mathcal{L}$, the first letter of $v$ is a 1 . Since $\hat{u}$ has period $2^{s}$, by Lemma 1 no subword of $\mu^{s}(01)$ of length greater than $2^{s}$ occurs in $\hat{u}$. We conclude that either $\hat{u}$ is a subword of $\delta^{t} \mu^{s}\left(0^{r}\right)$, or of $\mu^{s}(v)$, and hence of $\mu^{s}\left(0^{r-1} v\right)$. As this second case has been ruled out earlier, we conclude that $|\hat{u}| \leq\left|\delta^{t} \mu^{s}\left(0^{r}\right)\right|=r 2^{s}-t$. This gives a contradiction: $\hat{u}$ is a $\kappa$-power, yet $|\hat{u}| / q \leq\left(r 2^{s}-t\right) / 2^{s}=\beta<\alpha$.

By construction, $\delta^{t} \mu^{s}\left(0^{r} v\right)$ has the form $00 \hat{v}$ where $00 \hat{v} \in \mathcal{L}$.

We are now ready to prove our main theorem:

Theorem 6. Let $\alpha>2$ be a real number. There is a word over $\{0,1\}$ with critical exponent $\alpha$.

Proof. Call a real number $\beta<\alpha$ obtainable if $\beta$ can be written $\beta=r-t / 2^{s}$, where $r, s, t$ are positive integers, $s \geq 3$, and the word obtained by removing a prefix of length $t$ from $\mu^{s}(0)$ begins with 00 . We note that $\mu^{3}(0)=01101001$ and $\mu^{3}(1)=10010110$ are of length 8, and both contain 00 as a subword; for a given $s \geq 3$ it follows that $r$ and $t$ can be chosen so that $\beta=r-t / 2^{s}<\alpha$ and $|\alpha-\beta| \leq 7 / 2^{s}$; by choosing large enough $s$, an obtainable number $\beta$ can be chosen arbitrarily close to $\alpha$.

Let $\left\{\beta_{i}\right\}$ be a sequence of obtainable numbers converging to $\alpha$. For each $i$ write $\beta_{i}=r_{i}-t_{i} / 2^{s_{i}}$, where $r_{i}, s_{i}, t_{i}$ are positive integers, $s_{i} \geq 3$, and the word obtained by 
removing a prefix of length $t_{i}$ from $\mu^{s_{i}}(0)$ begins with 00 . If $00 w \in \mathcal{L}$, denote by $\phi_{i}(w)$ the word $\delta^{t_{i}} \mu^{s_{i}}\left(0^{r_{i}} w\right)$.

Consider the sequence of words

$$
\begin{aligned}
w_{1} & =\phi_{1}(\epsilon) \\
w_{2} & =\phi_{1}\left(\phi_{2}(\epsilon)\right) \\
w_{3} & =\phi_{1}\left(\phi_{2}\left(\phi_{3}(\epsilon)\right)\right) \\
& \vdots \\
w_{n} & =\phi_{1}\left(\phi_{2}\left(\phi_{3}\left(\cdots\left(\phi_{n}(\epsilon)\right) \cdots\right)\right)\right)
\end{aligned}
$$

By the third part of Lemma 5 , if $00 w \in \mathcal{L}$ is $\alpha$-power-free, then so is $\phi_{i}(w)$. Since $00 \epsilon$ is $\alpha$-power-free, each $w_{i}$ is therefore $\alpha$-power-free.

By the first and second parts of Lemma $5, w_{n}$ contains $\beta_{i}$-powers, $i=1,2, \ldots, n$.

Note that $\epsilon$ is a prefix of $\phi_{n+1}(\epsilon)$, so that

$$
w_{n}=\phi_{1}\left(\phi_{2}\left(\phi_{3}\left(\cdots\left(\phi_{n}(\epsilon)\right) \cdots\right)\right)\right)
$$

is a prefix of

$$
\phi_{1}\left(\phi_{2}\left(\phi_{3}\left(\cdots\left(\phi_{n}\left(\phi_{n+1}(\epsilon)\right)\right) \cdots\right)\right)\right)=w_{n+1} .
$$

We may therefore let $w=\lim _{n \rightarrow \infty} w_{i}$.

Since every prefix of $w$ is $\alpha$-power-free, $w$ is $\alpha$-power-free but contains $\beta_{i}$-powers for each $i$. The critical exponent of $w$ is therefore $\alpha$.

The following question raised by Krieger and Shallit remains open: for $\alpha>1$, if $\alpha$-powers are avoidable on a $k$-letter alphabet, does there exist an infinite word over $k$ letters with critical exponent $\alpha$ ? In particular, for $\alpha>\operatorname{RT}(k)$, where $\mathrm{RT}(k)$ denotes the repetition threshold on $k$ letters (see [2]), does there exist an infinite word over $k$ letters with critical exponent $\alpha$ ? We believe that the answer is "yes".

\section{Acknowledgments}

We would like to thank the anonymous referee for helpful comments and suggestions.

\section{References}

[1] F.-J. Brandenburg, "Uniformly growing $k$-th power-free homomorphisms", Theoret. Comput. Sci. 23 (1983), 69-82.

[2] A. Carpi, "On Dejean's conjecture over large alphabets", Theoret. Comput. Sci. 385 (2007), 137-151. 
[3] J.D. Currie, N. Rampersad, J. Shallit, "Binary words containing infinitely many overlaps", Electron. J. Combin. 13 (2006), \#R82.

[4] J. Karhumäki, J. Shallit, "Polynomial versus exponential growth in repetition-free binary words", J. Combin. Theory Ser. A 104 (2004), 335-347.

[5] D. Krieger, "On critical exponents in fixed points of binary $k$-uniform morphisms". In Proc. STACS 2006, LNCS 3884, Springer-Verlag, 2006, pp. 104-114.

[6] D. Krieger, "On critical exponents in fixed points of non-erasing morphisms", Theoret. Comput. Sci. 376 (2007), 70-88.

[7] D. Krieger, J. Shallit, "Every real number greater than 1 is a critical exponent", Theoret. Comput. Sci. 381 (2007), 177-182.

[8] F. Mignosi, G. Pirillo, "Repetitions in the Fibonacci infinite word", RAIRO Inform. Theor. Appl. 26 (1992), 199-204.

[9] A. M. Shur, "The structure of the set of cube-free $\mathbb{Z}$-words in a two-letter alphabet" (Russian), Izv. Ross. Akad. Nauk Ser. Mat. 64 (2000), 201-224. English translation in Izv. Math. 64 (2000), 847-871.

[10] D. Vandeth, "Sturmian words and words with a critical exponent", Theoret. Comput. Sci. 242 (2000), 283-300. 